\title{
Pneumonia
}

\section{Treatment and Diagnosis}

\author{
Joshua T. Mattila ${ }^{1}$, Michael J. Fine ${ }^{2,3}$, Andrew H. Limper ${ }^{4}$, Patrick R. Murray ${ }^{5}$, Bill B. Chen ${ }^{6,7}$, and Philana Ling Lin ${ }^{8}$ \\ ${ }^{1}$ Department of Microbiology and Molecular Genetics, ${ }^{2}$ Division of General Internal Medicine and ${ }^{6}$ Acute Lung Injury Center of \\ Excellence, ${ }^{7}$ Department of Medicine, University of Pittsburgh, Pittsburgh, Pennsylvania; ${ }^{3}$ VA Center for Health Equity Research and \\ Promotion, VA Pittsburgh Healthcare System, Pittsburgh, Pennsylvania; 'Division of Pulmonary and Critical Care, Department of Internal \\ Medicine, Mayo Clinic College of Medicine, Mayo Clinic, Rochester, Minnesota; ${ }^{5}$ Becton Dickinson Diagnostic Systems, Hunt Valley, \\ Maryland; and ${ }^{8}$ Department of Pediatrics, Children's Hospital of Pittsburgh of the University of Pittsburgh Medical Center, University of \\ Pittsburgh School of Medicine, Pittsburgh, Pennsylvania
}

\begin{abstract}
Pneumonia remains a leading cause of morbidity and mortality despite advances in treatment and therapy. The "Pneumonia: Treatment and Diagnosis" session of the Pittsburgh International Lung Conference examined topics related to improving care of patients with pneumonia. These topics included the process and quality of care for community-acquired pneumonia (CAP), diagnosis and treatment of emerging fungal pathogens, an overview of the strengths and weaknesses of different diagnostic modalities, and an example of how basic science is exploring immunomodulatory strategies for pneumonia treatment. Systematic health care provider and institutional improvements can decrease mortality rates in CAP, particularly in patients with increasingly complex comorbidities. Aspects of current guidelines for the diagnosis and treatment of fungal pneumonia were reviewed through a series of case
\end{abstract}

presentations. Proper treatment of pneumonia hinges on correct pathogen identification but is complicated by the variety of diagnostic assays with variable specificity, sensitivity, and interpretation. In addressing this topic, Dr. Patrick Murray, Ph.D., discussed a range of diagnostic tests for a variety of pathogens and guidelines for their use. In addition to the current state of CAP treatment, Bill (Beibei) Chen, M.D., Ph.D., presented a new potential therapeutic agent called forsythin, an immunomodulatory compound derived from a plant used in traditional Chinese medicine. These topics, ranging from institution-sized policy to interactions at the molecular scale, paint a broad perspective of the efforts against pneumonia.

Keywords: community-acquired infections; health care quality, access, and evaluation; mycoses; diagnostic techniques and procedures; inflammation mediators

(Received in original form January 22, 2014; accepted in final form March 7, 2014)

Author Contributions: J.T.M. and P.L.L. wrote the manuscript; M.J.F., A.H.L., P.R.M., and B.B.C. were conference presenters and reviewed their relevant sections; P.L.L. moderated the presentations.

Correspondence and requests for reprints should be addressed to Philana Ling Lin, M.D., M.Sci., One Children's Hospital Drive, 4401 Penn Avenue, Suite Floor 9, Pittsburgh, PA 15224. E-mail: Philana.Lin@chp.edu

Ann Am Thorac Soc Vol 11, Supplement 4, pp S189-S192, Aug 2014

Published 2014 by the American Thoracic Society

DOI: 10.1513/AnnalsATS.201401-027PL

Internet address: www.atsjournals.org

Pneumonia is a leading cause of hospitalization and mortality in the United States, especially among elderly and immunosuppressed populations. The Pittsburgh International Lung conference included a session focusing on this topic. A variety of topics were addressed by the presenters and ranged from the issues associated with institution-level quality of care to intracellular enzymes mediating inflammation. The speakers included Dr. Michael Fine (University of Pittsburgh School of Medicine), Dr. Andrew Limper (Mayo Clinic School of Medicine), Dr. Patrick Murray (Becton Dickinson Diagnostic Systems), and Dr. Beibei (Bill) Chen (University of Pittsburgh School of Medicine).

\section{Quality of Care for Community- acquired Pneumonia in the Twenty-First Century}

During the twentieth century, improvements in public health infrastructure leading to safer drinking water and technological advances, including the discovery of antibiotics to treat bacterial infections and development of vaccines against influenza and Streptococcus pneumoniae, played important roles in transforming pneumonia from the first to the sixth leading cause of death in the United States (1). Quality of medical care has further improved the medical outcomes of patients with this common medical illness over the past 2 decades. Quality of 
care can be defined as medical care that is expected to maximize an inclusive measure of patient welfare (2) and the degree to which health services for individuals and populations increase the likelihood of a desired outcome and are consistent with current professional knowledge (3). Based on a conceptual model proposed by Avedis Donabedian (2), quality of care can be measured using three key dimensions: structure of care (i.e., how medical care is organized at a health care facility or organizational level), processes of care (i.e., the actual diagnostic and/or therapeutic services that medical providers carry out as part of patient care), and outcomes of care (i.e., patient-centered medical outcomes). Improvements at all three levels of care have been responsible for the decline in CAP-associated mortality.

Beginning in the early 1990s, Medicare and The Joint Commission initiated collaborative national pneumonia quality improvement efforts that focused on tracking and eventually publically reporting performance rates for select evidence-based processes of care for patients hospitalized with pneumonia. Performance rates have dramatically improved over the past 2 decades, with current rates exceeding 95\% for assessment of arterial oxygenation, performing blood cultures in the emergency department and for patients admitted to an intensive care unit (ICU), rapid initiation and appropriate selection of initial antibiotic therapy, and immunization for influenza and pneumococcus. Although these gains are encouraging, there remains room for improvement. For example, appropriate initial antibiotic therapy for patients admitted to an ICU has lagged behind, with only $76 \%$ of such patients satisfying this measure in 2010.

Over the past 2 decades, short-term mortality for patients hospitalized for pneumonia has continued to decline, despite the fact that hospitalized patients have an increased burden of comorbid illnesses and a larger proportion are admitted to an ICU within a day of admission. Although it is not possible to demonstrate a causal association between improvements in the performance rates of evidence-based processes of care for pneumonia and the declining mortality from this illness, these changes in processes and outcomes allow one to conclude that we are advancing the needle for quality of care for patients with pneumonia thus far in the twenty-first century. Making continued improvements in the future will require several steps: continued public tracking and reporting of performance rates of pneumonia, processes of care, and patient outcomes; incentivizing hospitals and payers for performance on process and outcome quality metrics; addressing existing gaps in high-quality care (e.g., improving appropriate antibiotic therapy for ICU patients); improving surveillance programs for new and emerging pathogens and changing patterns of resistance; and developing new diagnostic tests, treatment regimens, and vaccines for established and emerging pulmonary pathogens.

\section{Fungal Pneumonia Guidelines: The Outcome and the Process}

Given the increasing incidence of fungal pneumonia (4), the American Thoracic Society published guidelines on the treatment of fungal pneumonia in 2011 to summarize the more recent changes in diagnostic and therapeutic regimens that are clinically relevant to patient care (5). These guidelines include both common and uncommon fungal pathogens of the lung, the strength and quality of the available data from which the guidelines were derived, and the process followed to generate the guidelines. Geographic environment, history of prior antibiotic use, treatment with immune suppressive agents, and transplantation (e.g., organ or bone marrow transplantation) are important risk factors for fungal pneumonia. In general, lung biopsy and culture are required, but less invasive diagnostic modalities are needed.

Histoplasma capsulatum is a dimorphic fungus endemic to the Ohio, Missouri, and the Mississippi River valley and is also found in poultry waste and bat guano (6). Histoplasmosis, infection with $H$. capsulatum, is typically asymptomatic but can be a significant source of morbidity in HIV-infected people and individuals undergoing tumor necrosis factor (TNF) agonist therapy (6); individuals with underlying lung disease, including emphysema, are also at risk (5). Histoplasmosis can mimic a variety of conditions and should be considered in patients with the appropriate geographic exposure and risk factors. The presence of both skin and lung lesions are a unique feature of invasive histoplasma disease. In contrast to other lung pathogens, pleural effusions are not a common manifestation of histoplasmosis. Last, multiple diagnostic platforms may be required to make the diagnosis, given the limited sensitivity and specificity of individual tests. For example, both serologic and urinary antigen assays may be required to make the diagnosis of histoplasmosis, but results from these assays may take several weeks.

Amphotericin B (or its derivatives) and itraconazole with or without steroids remain mainstays of treatment.

Coccidioidomycosis is caused by infection by either Coccidioides posadasii or Coccidioides immitis, soil-dwelling fungi endemic to arid regions of the southwest United States, Mexico, and parts of Central and South America (7). Like histoplasmosis, most Coccidioides infections are asymptomatic, and the most susceptible populations include immunosuppressed individuals, including individuals receiving solid organ transplants $(5,7)$. Because coccidiomycosis can resemble CAP, geographic location and travel history are important considerations in making the correct diagnosis. Again, multiple diagnostic platforms may be required to make the diagnosis, including fungal culture, serology, and polymerase chain reaction. Fluconazole, itraconazole, and amphotericin B remain the standard of care, but newer drugs such as voriconazole and posaconazole may have efficacy against coccidiomycosis.

Pneumonia from filamentous fungi infections often pose a diagnostic and therapeutic dilemma. Infection with mucormycosis (including Rhizopus, Mucor, Rhizomucor, and Cunninghamella) has become an increasingly important source of infection for immunocompromised individuals $(5,8,9)$. Among the populations most susceptible include individuals with diabetic ketoacidosis, HIV infection, solid organ transplant, and hematologic disorders with profound neutropenia $(5,8,9)$. Mucormycosis can be distinguished from other filamentous fungi by differences in hyphal morphology that may be seen on tissue biopsy. Nucleic acid amplification methods are available but have demonstrated limited clinical usefulness thus far. Aspergillosis results from invasive infection from Aspergillus fumigatus and, less commonly, Aspergillus flavus, Aspergillus niger, and Aspergillus terreus. Patients at risk for aspergillosis 
include immunosuppressed populations, especially neutropenic individuals, but also a range of nontraditionally immunosuppressed patients including individuals with chronic obstructive pulmonary disease, previous tuberculosis, and alcoholism (10). Differentiating moldlike pathogens is complex, and with other organisms in this group, multiple diagnostic modalities are often required. Less invasive antigen detection methods, such as galactomannan, have shown promise and can be helpful, but the accuracy of the assays still warrants further studies (e.g., administration of piperacillintazobactam has been known to be associated with false positives, but more recently this has been less of an issue with better antibiotic processing). Voriconazole has become the mainstay of treatment for invasive infections caused by Aspergillus spp. as well as amphotericin. Echinocandins are used primarily in salvage treatment.

\section{Emerging Diagnostic Techniques: Bacteria, Viruses, and Fungi}

Optimal treatment of lung infections relies on rapid and accurate detection of the offending pathogen. Delay in diagnosis can lead to increased morbidity and mortality. Factors that contribute to delayed diagnosis can include limitations in obtaining the appropriate culture specimen as well as suboptimal diagnostic techniques. The advantages and disadvantages of traditional and newly emerging techniques are discussed.

Culture remains the gold standard for identification of bacterial pathogens that cause lung infections. These methods are time consuming, because results are dependent on bacterial growth. Gram stain identification is susceptible to human error, as it is dependent on the expertise of the reader. Importantly, some types of bacteria require special media and conditions for optimal growth. Antigen detection techniques can lack sensitivity and specificity (e.g., Legionella antigen assays do not detect all serotypes). Nucleic acid amplification techniques (NAAT) for pathogen detection and identification are rapidly becoming mainstream diagnostic tests because they can return results in hours rather than days for culture-based techniques (if the organism in question can be cultured at all) and because of their high degree of sensitivity and specificity. Unfortunately, the high level of sensitivity for NAAT also makes them susceptible to high false-positive rates; therefore, caution is urged when making decisions solely based on their results.

Historic and emerging diagnostic tests for bacteria, Nocardia spp., Mycobacterium spp., and fungal and viral pathogens have different strengths and weaknesses. In the case of Nocardia, standard cultures should be held for longer periods of time. Buffered charcoal yeast extract agar is optimal for Nocardia growth and should be used on all bronchoalveolar lavage samples in which Legionella or Nocardia infection are considered. In the case of Mycobacterium tuberculosis, rapid and specific identification are of critical importance. Culture remains the gold standard method of identification but requires long incubation periods. NAAT offers the potential for rapid diagnosis as well as detection of drug sensitivity. The GeneXpert system (Cepheid, Sunnyvale, CA), an NAAT to detect $M$. tuberculosis and drug resistance, is now available in developing countries with high sensitivity among smear-positive samples. This assay has great point-of-care potential as it is cheap (estimated at \$10-15 U.S. dollars per assay when subsidized), rapid (results available within several hours), and requires far less labor and laboratory standardization compared with traditional methods of acidfast staining and culture of M. tuberculosis. This test has recently been U.S. Food and Drug Administration-approved for use in the United States.

Culture remains the gold standard for fungal identification as well. Antigen detection techniques (e.g., galactomannan for Aspergillus) are promising, but assay "cut-offs" still need to be reviewed for optimal sensitivity and specificity. Antibody detection and NAAT methods are not available. In the case of viruses, culture techniques including shell vial assays are largely outdated. Viral antigen assays provide rapid results but often have limited sensitivity (e.g., influenza A rapid antigen assays have $\sim 65 \%$ sensitivity), although the new generation of rapid immunoassay tests are significantly better. Multiplex NAAT platforms are currently in clinical use and are able to detect multiple viral pathogens simultaneously with high sensitivity and specificity. The advent of matrix-assisted laser desorption ionization time of flight (MALDI-TOF) mass spectrometry is likely to revolutionize identification of both bacterial and fungal pathogens. Despite advanced methods of pathogen detection, it still remains critical to distinguish pathogen carriage/colonization from invasive disease with specific clinical scenarios.

\section{Regulation of Inflammatory Pathways in Pneumonia}

An excessive inflammatory response can cause significant damage to lung tissue and contribute to the pathology associated with pneumonia, influenza, Aspergillus, and asthma. A variety of approaches have been applied to limit these responses, including inhibition of phosphodiesterase 4 (PDE4). PDE4 is abundant in immune cells and catalyzes cyclic AMP (cAMP), a second messenger associated with down-regulated proinflammatory activity (11). Decreased intracellular cAMP levels are associated with diminished $\mathrm{T}$ cell cytokine expression and proliferation, decreased superoxide production by eosinophils and neutrophils, inhibited elastase secretion by neutrophils, and inhibited TNF release by monocytes, macrophages, and dendritic cells (12). Although PDE4 inhibition has been considered as a means to control inflammatory responses, the pharmacologic inhibitors that are available in the clinic have problems, including limited efficacy and significant side effects that limit their usefulness. Traditional Chinese medicine has used seeds from the forsythia plant, a shrub in the olive family, to treat respiratory infections, suggesting this plant produces compounds with pharmacologic activity. Isolation of the components of the seeds has identified a molecule, forsythin, which may play a role. Structural analysis of forsythin indicates that it has a high degree of specificity for PDE4 and may be partly responsible for the forsythia seed's antiinflammatory activity. In vitro tests on human peripheral blood mononuclear cells indicate forsythin can inhibit cytokine expression. Moreover, experiments using a murine model of LPS-induced lung damage and bacterial pneumonia found less inflammation in forsythin-treated animals relative to untreated animals. These data suggest that forsythin may be a specific, functional inhibitor of PDE4 that has promise as an antiinflammatory therapeutic 
for treatment of inflammatory lung diseases.

\section{Conclusions}

Despite advances in treatment, diagnosis, and prevention, pneumonia remains a major cause of morbidity and mortality worldwide. This session provided a broad range of clinical, diagnostic, and immune modulatory perspectives regarding pneumonia. Great improvements in the treatment of CAP have been achieved through systemic changes at organizational and health care provider levels. Advances in the quality of care may be achieved by providing incentives for better-quality metrics, addressing gaps in health care, and improving diagnostic methods. The diagnosis of fungal lung infection remains difficult, often requiring multiple modalities, and new assays continue to be developed. Major improvements in identification of bacterial and viral pathogens will be available in the near future, although important considerations must be made in distinguishing colonization and infection, particularly in the context of the microbiome. Finally, a better understanding of lung inflammation in the context of infection is needed. In the future, immune modulation may play a critical role in the treatment of lung infections.

Author disclosures are available with the text of this article at www.atsjournals.org.

\section{References}

1 Center for Disease Control and Prevention (CDC). Achievements in public health, 1900-1999: control of infectious diseases. MMWR Morb Mortal Wkly Rep 1999;48:621-629.

2 Donabedian A. The quality of care: how can it be assessed? JAMA 1988;260:1743-1748.

3 Lohr KL, editor. Medicare: a strategy for quality assurance, volume I. Washington, DC: The National Academies Press; 1990.

4 Limper $\mathrm{AH}$. The changing spectrum of fungal infections in pulmonary and critical care practice: clinical approach to diagnosis. Proc Am Thorac Soc 2010;7:163-168.

5 Limper AH, Knox KS, Sarosi GA, Ampel NM, Bennett JE, Catanzaro A, Davies SF, Dismukes WE, Hage CA, Marr KA, et al.; American Thoracic Society Fungal Working Group. An official American Thoracic Society statement: treatment of fungal infections in adult pulmonary and critical care patients. Am J Respir Crit Care Med 2011;183:96-128.
6 McKinsey DS, McKinsey JP. Pulmonary histoplasmosis. Semin Respir Crit Care Med 2011;32:735-744.

7 Thompson GR III. Pulmonary coccidioidomycosis. Semin Respir Crit Care Med 2011;32:754-763.

8 Sun HY, Singh N. Mucormycosis: its contemporary face and management strategies. Lancet Infect Dis 2011;11:301-311.

9 Hamilos G, Samonis G, Kontoyiannis DP. Pulmonary mucormycosis. Semin Respir Crit Care Med 2011;32:693-702.

10 Kousha M, Tadi R, Soubani AO. Pulmonary aspergillosis: a clinical review. Eur Respir Rev 2011;20:156-174.

11 Beghè B, Rabe KF, Fabbri LM. Phosphodiesterase-4 inhibitor therapy for lung diseases. Am J Respir Crit Care Med 2013;188: 271-278.

12 Page CP, Spina D. Selective PDE inhibitors as novel treatments for respiratory diseases. Curr Opin Pharmacol 2012;12: 275-286. 